\title{
Phase-field modeling and simulation of fracture in brittle materials with strongly anisotropic surface energy
}

\author{
Bin Li, Christian Peco, Daniel Millán, Irene Arias and Marino Arroyo*, \\ Laboratori de Càlcul Numèric (LaCaN), Universitat Politècnica de Catalunya-BarcelonaTech (UPC), Barcelona, Spain
}

\begin{abstract}
SUMMARY
Crack propagation in brittle materials with anisotropic surface energy is important in applications involving single crystals, extruded polymers, or geological and organic materials. Furthermore, when this anisotropy is strong, the phenomenology of crack propagation becomes very rich, with forbidden crack propagation directions or complex sawtooth crack patterns. This problem interrogates fundamental issues in fracture mechanics, including the principles behind the selection of crack direction. Here, we propose a variational phase-field model for strongly anisotropic fracture, which resorts to the extended Cahn-Hilliard framework proposed in the context of crystal growth. Previous phase-field models for anisotropic fracture were formulated in a framework only allowing for weak anisotropy. We implement numerically our higher-order phase-field model with smooth local maximum entropy approximants in a direct Galerkin method. The numerical results exhibit all the features of strongly anisotropic fracture and reproduce strikingly well recent experimental observations. Copyright (C) 2014 John Wiley \& Sons, Ltd.
\end{abstract}

Received 1 May 2014; Revised 23 May 2014; Accepted 26 May 2014

KEY WORDS: fracture; meshfree methods; phase-field models; strongly anisotropic surface energy; local maximum entropy approximants

\section{INTRODUCTION}

Most materials are anisotropic, also with regard to their fracture behavior. While most theoretical and computational studies have focused on elastic anisotropy [1-3], the anisotropy of the fracture toughness influences more strongly the crack propagation of a wide variety of materials including single crystals [4-6], extruded polymers [7], geological materials [8, 9], including sedimentary [10] and granitic rocks [11], or apple flesh [12]. The issue of brittle crack propagation in materials with anisotropic surface energy deeply interrogates our understanding of fracture and is receiving increasing attention from a variety of points of view, such as molecular dynamics [13], continuum mechanics [14, 15], phase-field modeling [16, 17], and experiments [7, 18]. Here, by exploiting the analogy with crystal growth, we develop and implement numerically a phase-field model for brittle fracture of materials with strongly anisotropic surface energy and interpret our numerical results in the light of recent theories and experiments.

The current theoretical framework for brittle fracture was initiated nearly a century ago by Griffith [19]. In this theory, crack propagation arises as a balance between the surface energy and the release of elastic energy; a crack will propagate in a direction given by the angle $\theta$ when the relation

$$
G(\theta)=G_{c}
$$

*Correspondence to: Marino Arroyo, Laboratori de Càlcul Numèric (LaCaN), Universitat Politècnica de CatalunyaBarcelonaTech (UPC), Barcelona, Spain.

†E-mail: marino.arroyo@upc.edu 
holds, where $G(\theta)$ is the elastic energy release rate for a crack along $\theta$ and $G_{c}$ is the surface energy of the newly created crack faces. While this theory has proved to be very useful to describe the various features of cracks [20], it does not address the important question of how to determine the crack path, that is, the angle $\theta$. Under quasi-static loading, several popular criteria have been appended to Griffith's theory to determine the crack path, including (1) the principle of local symmetry [21, 22]; (2) the maximum energy release rate (MERR) [14, 21, 23]; (3) the minimum strain energy density [24]; and (4) the maximum hoop stress [25]. While these criteria provide similar predictions for homogeneous isotropic materials (in fact, (1) and (2) coincide under certain conditions [15, 26]), they greatly differ when generalized to materials with anisotropic surface energy, in which the fracture toughness $G_{c}(\theta)$ is orientation dependent. When generalizing MERR, Griffith's propagation Equation (1) is first met along a direction $\theta$ such that $G(\theta) / G_{c}(\theta)$ is maximized [7, 15], which provides a criterion to select the crack direction. If these functions are smooth and recalling Equation (1), the optimality condition results in $d G / d \theta=d G_{c} / d \theta$, which can be identified as a configurational torque balance $[16,17]$.

Recent experiments on thin anisotropic films have interrogated the MERR criterion in materials with anisotropic fracture toughness, finding results inconsistent with the global maximization of $G(\theta) / G_{c}(\theta)$ [7]. By examining strongly anisotropic materials, these experiments established a close analogy with crystal growth and the Wulff construction for the equilibrium shape of a crystal in materials with strongly anisotropic surface energy, which exhibit forbidden orientations and faceted surfaces. This analogy had been previously pointed out theoretically in [14]. Furthermore, these experiments report crack propagation along metastable directions, suggesting a principle based on local maximization rather than global maximization of $G(\theta) / G_{c}(\theta)$.

While phase-field models have been central in these investigations [16, 17], they have been developed within a framework that only allows for weakly twofold anisotropic surface energies (elliptic polar energy graphs). However, many of the interesting features of fracture in anisotropic materials, such as sawtooth crack patterns or forbidden crack directions, are directly related to the nonconvexity of strongly anisotropic surface energies [7]. Here, we start from a regularized variational theory of brittle fracture [27] and modify it by formulating a strongly anisotropic surface energy inspired by phase-field models of crystal growth. This results in a fourth-order system of partial differential equations (PDEs) for the displacement and for the phase-field representing the cracks. The variational nature of this model suggests that the underlying crack-path selection principle is related to the MERR criterion, and in fact, it has been shown through asymptotic analysis that cracks propagate obeying the configurational equilibrium $d G / d \theta=d G_{c} / d \theta$ in a weakly anisotropic phase-field model [17]. We implement numerically the proposed model with local maximum entropy (LME) meshfree approximants [28], which allow us to directly deal with the high-order nature of the PDE $[29,30]$, and explore the fracture behavior of the model in the light of the experiments by [7].

In Section 2, we summarize previous variational phase-field models for fracture, introduce the notion of the extended Cahn-Hilliard $(\mathrm{ECH})$ model to define anisotropic surface energies, and show how to integrate it in a model for fracture. Section 3 succinctly describes the numerical implementation of the model, and Section 4 presents representative simulations, showing the fundamental features of fracture in materials with strongly anisotropic surface energy. Section 5 collects our final remarks and conclusions.

\section{PHASE-FIELD MODEL FOR MATERIALS WITH ANISOTROPIC SURFACE ENERGY}

\subsection{Background}

A variational free-discontinuity generalization of Griffith's theory of brittle fracture was proposed by Francfort and Marigo [31], addressing issues such as crack nucleation, path selection, and discontinuous crack propagation. In this theory, the total energy, including bulk elastic and crack surface contributions, is simultaneously minimized with respect to any admissible crack set and displacement field. This theory was subsequently regularized into a phase-field model, suitable for numerical calculations [27], and which converges to the sharp variational theory of brittle fracture [32]. These works have prompted a large body of literature in mathematics, mechanics, and computational 
mechanics that we do not attempt to review here. In the regularized model, cracks are represented by a phase-field variable (scalar order parameter) $\phi$, which is 0 inside a cracked zone, 1 away from the crack, and changes from 0 to 1 smoothly. The total free energy of a possibly cracked elastic body $\Omega$ is modeled by

$$
E[\mathbf{u}, \phi]=\int_{\Omega}\left(\phi^{2}+\eta_{k}\right) W(\varepsilon) d \Omega+\int_{\Omega} G_{c}\left[\frac{(\phi-1)^{2}}{4 \kappa}+\kappa|\nabla \phi|^{2}\right] d \Omega
$$

where $\mathbf{u}$ is the displacement field, $W$ is the elastic strain energy depending on the strain tensor $\boldsymbol{\varepsilon}=1 / 2\left(\nabla \mathbf{u}+\nabla \mathbf{u}^{T}\right)$, and $G_{c}$ is the energy release rate (fracture surface energy). This family of functionals is parametrized by $\kappa>0$, a regularization parameter with units of length dictating the width of the smeared crack. When it goes to zero, the regularized model converges to the Griffithlike model studied in [31], but numerical simulations require a finite value of $\kappa$, which needs to be resolved by the grid. The parameter $\eta_{k}$ is such that $0<\eta_{k}<<1$ and can be seen as a vanishing residual stiffness of the cracks. Although it is not technically necessary from a mathematical standpoint [33], it is used to prevent ill-conditioning of stiffness matrix in the numerical implementation. For simplicity, we do not consider here body forces or surface tractions. The first integral of the functional is the elastic energy of a possibly damaged material, while the second integral approximates the surface energy. The minimization of the functional in Equation (2), with respect to both $\mathbf{u}$ and $\phi$, subject to Dirichlet data and to irreversibility of cracks, provides a computable approximation of the generalized Griffith's fracture theory.

Because the Euler-Lagrange equations resulting from Equation (2) is a system of second-order PDE, it has been referred to as a second-order phase-field theory of fracture [34]. The profile of the phase-field perpendicular to the crack can be analyzed by neglecting the elastic energy and looking for a one-dimensional stationary solution. As shown in Figure 1(a), the second-order phase-field model leads to a $C^{0}$ solution, exhibiting a discontinuous derivative at the crack. Because greater regularity of the exact solution provides better accuracy and convergence rates for numerical solutions, a higher-order phase-field model was introduced [34], where total free energy is given by

$$
E[\mathbf{u}, \phi]=\int_{\Omega}\left(\phi^{2}+\eta_{k}\right) W(\mathbf{u}) d \Omega+\int_{\Omega} G_{c}\left[\frac{(\phi-1)^{2}}{4 \kappa}+\frac{1}{2} \kappa|\nabla \phi|^{2}+\frac{1}{4} \kappa^{3}(\Delta \phi)^{2}\right] d \Omega
$$

This fourth-order phase-field theory leads to $C^{1}$ continuous solutions, as shown in Figure 1(b).

When turning to materials with anisotropic surface energy, the parameter $G_{c}$ in the model can no longer be a scalar because it needs to be orientation-dependent. Anisotropic surface energy is also very important in crystal growth or solidification, extensively studied in the materials science literature. Anisotropic surface energy has been specifically studied in the context of phase-field models for crystal growth/solidification. In a classical model [35], the coefficient pre-multiplying $|\nabla \phi|^{2}$ in

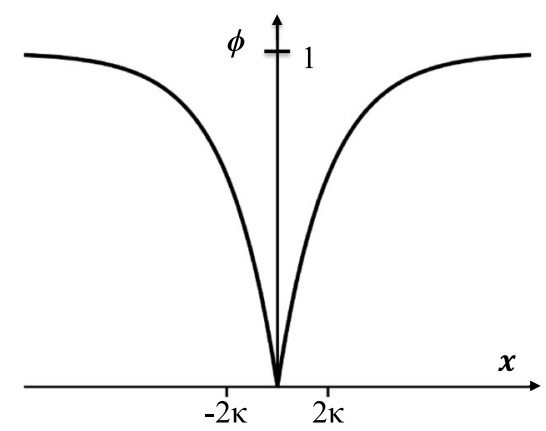

(a)

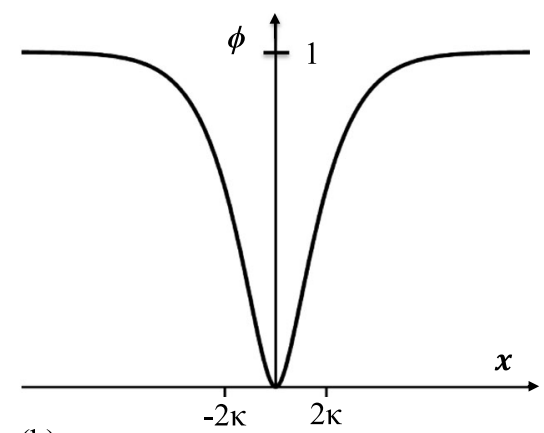

(b)

Figure 1. One-dimensional phase-field approximation of crack at $x=0$ : (a) second-order phase-field theory and (b) fourth-order phase-field theory. 
the surface energy is made dependent on the phase-field approximation of the outer normal vector $\mathbf{n}=\frac{\nabla \phi}{|\nabla \phi|}$ to the interface. In this way, such models introduce explicitly an orientation-dependent surface energy, $G_{c}(\mathbf{n})$ in our context. This approach is very appealing because it allows one to freely choose the functional form of this dependence. For strongly anisotropic surface energy, that is, when the polar plot of the reciprocal surface energy, $1 / G_{c}(\mathbf{n})$, is non-convex, it has been shown that the corresponding phase-field equations become ill-posed [36]. An expedite and homogenized approach to deal with strongly anisotropic surface energies is to convexify the surface energy [37], at the expense of missing interesting details of the geometric structure of the free-surface. Because strongly anisotropic surface energies are important in applications and the details about the freesurface matter, researchers have developed other remedies to this difficulty, for instance, regularizing the phase-field functional by adding the square of the Laplacian or a phase-field approximation to the Willmore curvature energy; see [38] and references therein. This method works well for models with phase-field variations across the interface approximating smoothly step functions, such as hyperbolic tangent profiles, but unfortunately cannot be used for phase-field model of fracture. The higher-order terms required to regularize the model result in a $C^{1}$ continuous phase-field for the crack as in Figure 1(b). At the center of the crack, $\nabla \phi=\mathbf{0}$, which renders the formula $\mathbf{n}=\frac{\nabla \phi}{|\nabla \phi|}$ inapplicable. One can try to remedy this problem by defining the outer normal vector $\mathbf{n}$ using eigenvectors of the Hessian matrix $\nabla^{2} \phi$, but this definition becomes problematic at other regions, particularly at the crack tip.

A different and natural way to take anisotropy into account is to follow the approach presented in the original work by Cahn and Hilliard [39] and write the Taylor series expansion of the free energy including higher-order terms. In the context of crystal growth, this idea has been shown to provide a satisfactory way of describing systems with anisotropic surface energy and is intrinsically regularized [40, 41]. However, as shown later, this method imposes constraints on the kinds of orientation dependence of $G_{c}(\mathbf{n})$. In this paper, we adapt this approach to fracture.

\subsection{Extended Cahn-Hilliard interface model}

The classical phase-field model for isotropic systems was developed in [39]. In this diffuse interface description, the behavior of a non-uniform system is characterized by the interfacial free energy $F$ and expressed as an integral of the local free energy density $f$ that is a function of the phase-field $\phi$. It can be expanded in a Taylor series about a given phase-field, provided that $f$ is a continuously differentiable function of its variables:

$$
\begin{aligned}
f\left(\phi, \nabla \phi, \nabla^{2} \phi, \ldots\right)= & f_{0}(\phi)+\sum_{i} L_{i} \frac{\partial \phi}{\partial x_{i}}+\sum_{i j} \kappa_{i j}^{(1)} \frac{\partial^{2} \phi}{\partial x_{i} \partial x_{j}} \\
& +\frac{1}{2} \sum_{i j} \kappa_{i j}^{(2)} \frac{\partial \phi}{\partial x_{i}} \frac{\partial \phi}{\partial x_{j}}+\ldots,
\end{aligned}
$$

where $\nabla$ and $\nabla^{2}$ are the gradient and the Hessian operators and

$$
\begin{gathered}
L_{i}=\left[\frac{\partial f}{\partial p_{i}}\right]_{0}, p_{i}=\frac{\partial \phi}{\partial x_{i}}, \\
\kappa_{i j}^{(1)}=\left[\frac{\partial f}{\partial q_{i j}}\right]_{0}, q_{i j}=\frac{\partial^{2} \phi}{\partial x_{i} \partial x_{j}}, \\
\kappa_{i j}^{(2)}=\left[\frac{\partial^{2} f}{\partial p_{i} \partial p_{j}}\right]_{0} .
\end{gathered}
$$


The subscript 0 indicates evaluation at a given phase-field. By applying the divergence theorem, the two second-rank tensor $\kappa_{i j}^{(1)}$ and $\kappa_{i j}^{(2)}$ can be reduce to only one [39]. Therefore, the interfacial free energy is given by

$$
F[\phi]=\int_{\Omega}\left(f_{0}(\phi)+\sum_{i} L_{i} \frac{\partial \phi}{\partial x_{i}}+\sum_{i j} \kappa_{i j} \frac{\partial \phi}{\partial x_{i}} \frac{\partial \phi}{\partial x_{j}}\right) d \Omega,
$$

where $\kappa_{i j}=-\frac{d \kappa_{i j}^{(1)}}{d \phi}+\frac{1}{2} \kappa_{i j}^{(2)}$. This model for the interfacial energy involves a second-rank tensor, $\kappa_{i j}$. Therefore, it can describe up to orthorhombic weak anisotropy, as exploited in previous phasefield models of anisotropic fracture [17]. But this anisotropy is too restrictive; for instance, it cannot describe the common cubic symmetry and cannot model strongly anisotropic effects [36]. Because higher-rank tensors can produce more general anisotropy, we extend the Taylor series expansion of local free energy density to higher order, here up to fourth order. This leads to ECH-type equations [40-42].

Following this approach, we expand the local free energy density $f$ to higher order

$$
\begin{aligned}
f\left(\phi, \nabla \phi, \nabla^{2} \phi, \ldots\right)= & f_{0}(\phi)+\sum_{i j} \kappa_{i j}^{(1)} \frac{\partial^{2} \phi}{\partial x_{i} \partial x_{j}}+\frac{1}{2} \sum_{i j} \kappa_{i j}^{(2)} \frac{\partial \phi}{\partial x_{i}} \frac{\partial \phi}{\partial x_{j}} \\
& +\frac{1}{24} \sum_{i j k l} \lambda_{i j k l}^{(1)} \frac{\partial \phi}{\partial x_{i}} \frac{\partial \phi}{\partial x_{j}} \frac{\partial \phi}{\partial x_{k}} \frac{\partial \phi}{\partial x_{l}}+\frac{1}{6} \sum_{i j k l} \lambda_{i j k l}^{(2)} \frac{\partial^{2} \phi}{\partial x_{i} \partial x_{j}} \frac{\partial \phi}{\partial x_{k}} \frac{\partial \phi}{\partial x_{l}} \\
& +\frac{1}{2} \sum_{i j k l} \lambda_{i j k l}^{(3)} \frac{\partial^{2} \phi}{\partial x_{i} \partial x_{j}} \frac{\partial^{2} \phi}{\partial x_{k} \partial x_{l}}+\frac{1}{2} \sum_{i j k l} \lambda_{i j k l}^{(4)} \frac{\partial^{3} \phi}{\partial x_{i} \partial x_{j} \partial x_{k}} \frac{\partial \phi}{\partial x_{l}} \\
& +\frac{1}{2} \sum_{i j k l} \lambda_{i j k l}^{(5)} \frac{\partial^{4} \phi}{\partial x_{i} \partial x_{j} \partial x_{k} \partial x_{l}}+\ldots
\end{aligned}
$$

The tensorial Taylor series coefficients must reflect the underlying symmetry of material. We omit odd-ranked tensors, because they are zero for the centrosymmetric materials of interest in the present study. As in [39], we present in the following text a formal definition of the remaining even-ranked tensors:

$$
\begin{gathered}
\lambda_{i j k l}^{(1)}=\left[\frac{\partial^{4} f}{\partial p_{i} \partial p_{j} \partial p_{k} \partial p_{l}}\right]_{0}, \\
\lambda_{i j k l}^{(2)}=\left[\frac{\partial^{3} f}{\partial q_{i j} \partial p_{k} \partial p_{l}}\right]_{0}, \\
\lambda_{i j k l}^{(3)}=\left[\frac{\partial^{2} f}{\partial q_{i j} \partial q_{k l}}\right]_{0}, \\
\lambda_{i j k l}^{(4)}=\left[\frac{\partial^{2} f}{\partial r_{i j k} \partial p_{l}}\right]_{0}, r_{i j k}=\frac{\partial^{3} \phi}{\partial x_{i} \partial x_{j} \partial x_{k}}, \\
\lambda_{i j k l}^{(5)}=\left[\frac{\partial f}{\partial s_{i j k l}}\right]_{0}, s_{i j k l}=\frac{\partial^{4} \phi}{\partial x_{i} \partial x_{j} \partial x_{k} \partial x_{i}} .
\end{gathered}
$$


From these definitions and because the order of differentiation can be exchanged for sufficiently smooth functions, these tensors possess various symmetries. Following similar arguments as before, the number of fourth-rank tensors can be reduced from five to three [40], and hence, the local free energy density $f$ can be written as

$$
\begin{aligned}
f\left(\phi, \nabla \phi, \nabla^{2} \phi\right)= & f_{0}(\phi)+\sum_{i j} \kappa_{i j} \frac{\partial \phi}{\partial x_{i}} \frac{\partial \phi}{\partial x_{j}}+\sum_{i j k l} \tilde{\alpha}_{i j k l} \frac{\partial \phi}{\partial x_{i}} \frac{\partial \phi}{\partial x_{j}} \frac{\partial \phi}{\partial x_{k}} \frac{\partial \phi}{\partial x_{l}} \\
& +\sum_{i j k l} \tilde{\beta}_{i j k l} \frac{\partial^{2} \phi}{\partial x_{i} \partial x_{j}} \frac{\partial \phi}{\partial x_{k}} \frac{\partial \phi}{\partial x_{l}}+\sum_{i j k l} \tilde{\gamma}_{i j k l} \frac{\partial^{2} \phi}{\partial x_{i} \partial x_{j}} \frac{\partial^{2} \phi}{\partial x_{k} \partial x_{l}} .
\end{aligned}
$$

\subsection{Anisotropic phase-field fracture model}

Here, we only consider cubic symmetry, and therefore, the second-rank tensor $\kappa_{i j}$ should be isotropic, that is, a scalar coefficient $\kappa$, which as discussed earlier has units of length in the present context (Equation (2)). Under cubic symmetry, any given fourth-rank tensor $C_{i j k l}$ expressed in the material principal axes has only three independent parameters. Resorting to Voigt notation, such tensors in $3 \mathrm{D}$ take the form

$$
\left[\begin{array}{cccccc}
C_{11} & C_{12} & C_{12} & & & \\
C_{12} & C_{11} & C_{12} & & 0 & \\
C_{12} & C_{12} & C_{11} & & & \\
& & & C_{44} & & \\
& 0 & & & C_{44} & \\
& & & & & C_{44}
\end{array}\right]
$$

while in $2 \mathrm{D}$, the tensor $C_{i j k l}$ can be written as

$$
\left[\begin{array}{ccc}
C_{11} & C_{12} & 0 \\
C_{12} & C_{11} & 0 \\
0 & 0 & C_{44}
\end{array}\right]
$$

See Appendix A for the expression of these tensors when the principal material axes are not aligned with the coordinate axes.

By using this rule for $\tilde{\alpha}_{i j k l}, \tilde{\beta}_{i j k l}$, and $\tilde{\gamma}_{i j k l}$ and by rescaling these tensors as $(\alpha, \beta, \gamma)=$ $1 / \kappa^{3}(\tilde{\alpha}, \tilde{\beta}, \tilde{\gamma})$ to make them non-dimensional, the surface energy $F$ in 2D becomes

$$
\begin{aligned}
F[\phi]=\bar{G}_{c} \int_{\Omega}\left\{f_{0}(\phi)+\kappa|\nabla \phi|^{2}+\kappa^{3}\left[2\left(\alpha_{12}+2 \alpha_{44}-\alpha_{11}\right)\left(\frac{\partial \phi}{\partial x}\right)^{2}\left(\frac{\partial \phi}{\partial y}\right)^{2}\right.\right. \\
+\alpha_{11}|\nabla \phi|^{4}+\left(\beta_{12}-\beta_{11}\right)\left(\frac{\partial^{2} \phi}{\partial x^{2}}\left(\frac{\partial \phi}{\partial y}\right)^{2}+\frac{\partial^{2} \phi}{\partial y^{2}}\left(\frac{\partial \phi}{\partial x}\right)^{2}\right) \\
+4 \beta_{44} \frac{\partial^{2} \phi}{\partial x \partial y} \frac{\partial \phi}{\partial x} \frac{\partial \phi}{\partial y}+\beta_{11}|\nabla \phi|^{2} \Delta \phi+2\left(\gamma_{12}-\gamma_{11}\right) \frac{\partial^{2} \phi}{\partial x^{2}} \frac{\partial^{2} \phi}{\partial y^{2}} \\
\left.\left.+4 \gamma_{44}\left(\frac{\partial^{2} \phi}{\partial x \partial y}\right)^{2}+\gamma_{11}(\Delta \phi)^{2}\right]\right\} d \Omega,
\end{aligned}
$$

where $\bar{G}_{c}$ is an energy per unit area scale for the surface energy. As discussed in the following text, the actual surface energy $G_{c}(\theta)$ in this model is orientation dependent, and therefore, $\bar{G}_{c}$ does not have a direct mechanical interpretation. The 3D interfacial free energy can be written analogously. 
Having the aforementioned anisotropic interfacial free energy $F$, we can formulate the anisotropic phase-field model for fracture as

$$
E[\mathbf{u}, \phi]=\int_{\Omega}\left(g(\phi)+\eta_{k}\right) W(\varepsilon) d \Omega+F[\phi],
$$

where there are different choices for the functions $f_{0}(\phi)$ and $g(\phi)$ consistent with the variational theory of brittle fracture; see, for example, $[43,44]$ for a discussion. Here, we adopt the standard choices $f_{0}=(1-\phi)^{2} /(4 \kappa)$ and $g(\phi)=\phi^{2}$, although it has been suggested that $g(\phi)=\phi^{3}$ prevents the emergence of spurious damage away from the crack tip and better mimics a linear elastic-brittle behavior for finite $\kappa$ [45], at the expense of nonlinearity in the model.

\subsection{Resulting anisotropic surface energy}

To gain insight about the resulting anisotropic surface energy, we consider a planar crack interface with a normal vector $\mathbf{n}$ forming an angle $\theta$ with the $x$-axis and introduce a coordinate perpendicular to the crack $z=\mathbf{x} \cdot \mathbf{n}$. We neglect the elastic energy and rewrite the free energy in Equation (18), assuming that $\phi=\phi(z)$. We have $\frac{\partial \phi}{\partial x}=\frac{\partial \phi}{\partial z} \cos \theta$ and $\frac{\partial \phi}{\partial y}=\frac{\partial \phi}{\partial z} \sin \theta$, and consequently, the surface energy becomes

$$
\begin{aligned}
F[\phi]=\bar{G}_{c} \int_{\Omega}\{ & f_{0}(\phi)+\kappa\left(\frac{\partial \phi}{\partial z}\right)^{2}+\kappa^{3}\left[\left(\alpha_{11}+\frac{1}{2}\left(\alpha_{12}-\alpha_{11}+2 \alpha_{44}\right) \sin ^{2}(2 \theta)\right)\left(\frac{\partial \phi}{\partial z}\right)^{4}\right. \\
& +\left(\beta_{11}+\frac{1}{2}\left(\beta_{12}-\beta_{11}+2 \beta_{44}\right) \sin ^{2}(2 \theta)\right) \frac{\partial^{2} \phi}{\partial z^{2}}\left(\frac{\partial \phi}{\partial z}\right)^{2} \\
& \left.\left.+\left(\gamma_{11}+\frac{1}{2}\left(\gamma_{12}-\gamma_{11}+2 \gamma_{44}\right) \sin ^{2}(2 \theta)\right)\left(\frac{\partial^{2} \phi}{\partial z^{2}}\right)^{2}\right]\right\} d \Omega
\end{aligned}
$$

The tensor $\beta_{i j k l}$ has no contribution to the surface energy, because

$$
\begin{gathered}
\int_{\Omega}\left(\beta_{11}+\frac{1}{2}\left(\beta_{12}-\beta_{11}+2 \beta_{44}\right) \sin ^{2}(2 \theta)\right) \frac{\partial^{2} \phi}{\partial z^{2}}\left(\frac{\partial \phi}{\partial z}\right)^{2} d \Omega \\
=\left.\frac{1}{3}\left(\beta_{11}+\frac{1}{2}\left(\beta_{12}-\beta_{11}+2 \beta_{44}\right) \sin ^{2}(2 \theta)\right)\left(\frac{\partial \phi}{\partial z}\right)^{3}\right|_{-\infty} ^{+\infty}
\end{gathered}
$$

and $\partial \phi / \partial z$ vanishes away from the crack. Consequently, this term does not enter into the EulerLagrange equation in this idealized planar crack setting. Accordingly, the energy in Equation (20) simplifies to

$$
F[\phi]=\bar{G}_{c} \int_{\Omega}\left\{f_{0}(\phi)+\kappa\left(\frac{\partial \phi}{\partial z}\right)^{2}+\kappa^{3}\left[\alpha\left(\frac{\partial \phi}{\partial z}\right)^{4}+\gamma\left(\frac{\partial^{2} \phi}{\partial z^{2}}\right)^{2}\right]\right\} d \Omega,
$$

where $\gamma$ is given by

$$
\begin{gathered}
\gamma=\gamma_{0}\left(1+\gamma_{4} \cos (4 \theta)\right), \\
\gamma_{0}=\frac{3 \gamma_{11}+\gamma_{12}+2 \gamma_{44}}{4}, \gamma_{4}=\frac{\gamma_{11}-\gamma_{12}-2 \gamma_{44}}{3 \gamma_{11}+\gamma_{12}+2 \gamma_{44}},
\end{gathered}
$$

and $\alpha$ can be written similarly. 
In the absence of an analytical solution to the Euler-Lagrange equation of this functional, the crack profile and interface energy can be calculated numerically. To compute the surface energy as a function of orientation, $G_{c}(\theta)$, we fix $\theta$ and solve the Euler-Lagrange equation of the functional in Equation (22), a fourth-order ordinary differential equation, subject to the following boundary conditions: $\phi(0)=\phi^{\prime}(0)=\phi^{\prime}(+\infty)=0$ and $\phi(+\infty)=1$. In practice, one of the boundaries is taken sufficiently far away from $z=0$, for example, $50 \kappa$, and the differential equation is approximated with MATLAB's $b v p 4 c$ function, implementing an adaptive collocation method. For each $\theta$, we evaluate the functional in Equation (22) at corresponding optimal profile to obtain a polar plot of the surface energy and its reciprocal, as shown in Figure 2 for several parameter values. It is clear that consistently with the assumed cubic symmetry of the model, the polar plots exhibit fourfold symmetry. The figure shows that the tensor $\gamma_{i j k l}$ can produce both weakly and strongly anisotropic energies, as indicated by the convexity of the polar plots of $1 / G_{c}(\theta)$. Because the tensors $\alpha_{i j k l}$ and $\beta_{i j k l}$ introduce nonlinearity in the surface energy, which becomes non-quadratic, here we focus on models including only the tensor $\gamma_{i j k l}$. It is interesting to note that models with only the tensor $\alpha_{i j k l}$ can produce strongly anisotropic surface energies (not shown here) but result in nonlinear secondorder PDEs. This makes the numerical discretization simpler, as $C^{0}$ finite elements could be directly used, but as previously discussed, other second-order strongly anisotropic models have been shown to be ill-posed because the energy of kinks is not stabilized [36].

With Figure 2 at hand, we can further discuss the notion of strong surface energy anisotropy. The classical Wulff construction for the equilibrium shape of crystals [46] naturally distinguishes between energies with convex and non-convex reciprocal energy plot. Non-convex plots lead to forbidden free-surface directions and faceting. In the crystal growth literature, there is another local notion of surface stability, given in $2 \mathrm{D}$ by the surface stiffness

$$
S(\theta)=G_{c}^{\prime \prime}(\theta)+G_{c}(\theta)
$$
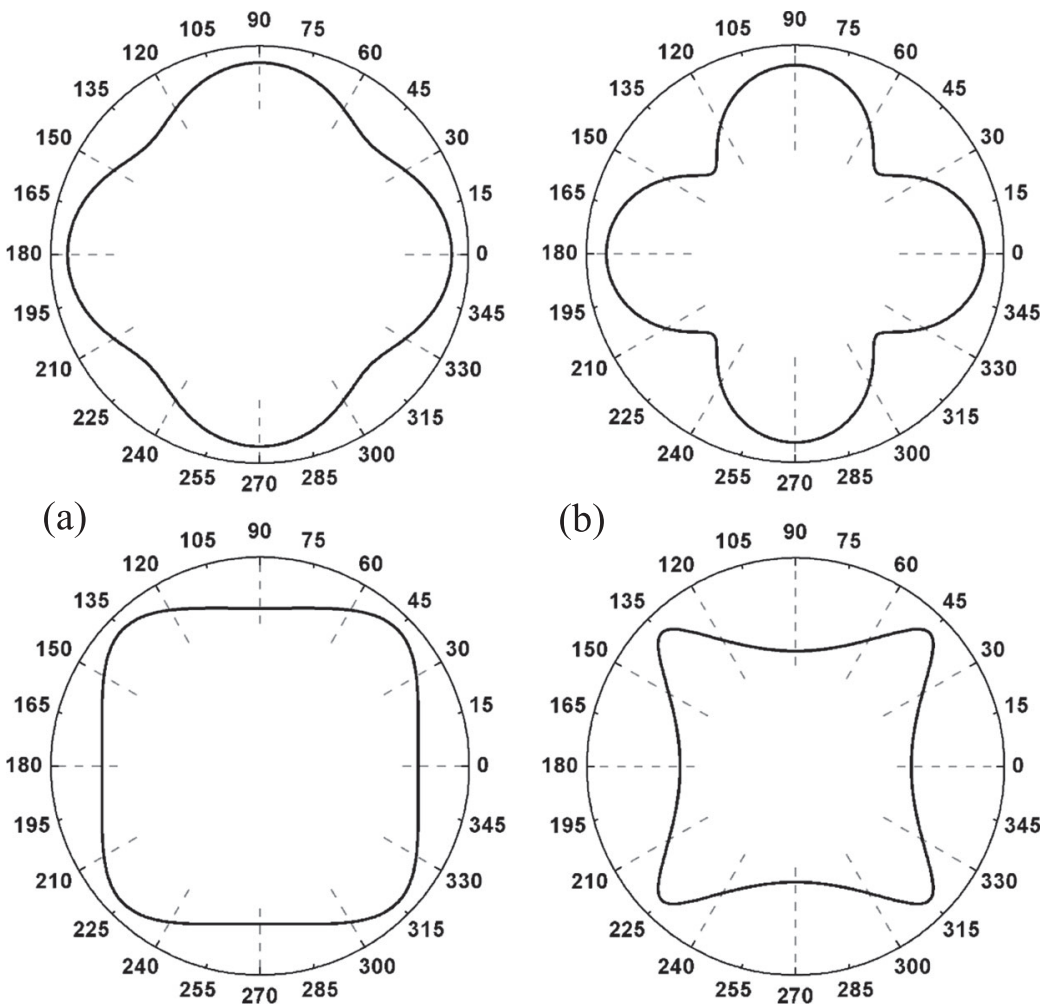

Figure 2. Polar representation of the surface energy (top) and its reciprocal (bottom) only considering the tensor $\gamma_{i j k l}$ ( $\alpha$ and $\beta$ are set to zero). (a) shows a weakly anisotropic energy (convex reciprocal energy plot) with $\gamma_{0}=10.0$ and $\gamma_{4}=0.5$. (b) shows a strongly anisotropic energy with $\gamma_{0}=10.0$ and $\gamma_{4}=0.9$. 
which has the sign of the curvature of the polar plot of the reciprocal surface energy [47]. This local characterization of convexity, and of strong/weak anisotropy, does not coincide with the global characterization in terms of the convexity of the polar plot of $1 / G_{c}(\theta)$ for non-smooth energies. Furthermore, as illustrated in the examples and in [7], the local characterization of free-surface (crack) stability is less restrictive than the global characterization, in that, it allows for metastable orientations. The local characterization of surface stability in 3D is discussed in [48].

We note that in the present phase-field model, the thickness of the interface depends on the orientation. Identifying the smallest thickness is important to set the parameter $\kappa$, with units of length, relative to the grid spacing. In the isotropic limit, this model gives rise to a family of higher-order phase-field models. In particular, if all terms $\alpha_{i j}, \beta_{i j}$, and $\gamma_{i j}$ are zero except for $\gamma_{11}=\gamma_{12}$, we recover the fourth-order model proposed in [34].

\section{NUMERICAL IMPLEMENTATION}

Because the free energy involves second-order derivatives of $\phi$, a direct Galerkin approach requires $C^{1}$ continuous approximations. We resort here to LME approximants [28], a meshfree method with non-negative and smooth basis functions. In this method, the support size of the basis functions can be modified through a non-dimensional aspect ratio parameter, which we take equal to 1.0 in all examples [49]. LME approximants have been successfully applied to fourth-order phasefield models, for example, in the simulation of biomembranes [30, 50]. Adaptive local refinement is straightforward and leads to very efficient phase-field solutions as elaborated in the references earlier, although we do not fully exploit this feature here. Adaptive refinement can be cumbersome in other techniques delivering smooth approximants, such as isogeometric methods. However, recent advances in T-splines [51] and hierarchical B-splines [52] alleviate the rigidity of these methods and may soon become accessible in 3D [53]. These adaptive spline techniques have been exploited in the numerical approximation of higher-order phase-field models of fracture [34]. To combine the highly accurate boundary representation of isogeometric methods with the flexibility of LME in the bulk, we have recently proposed a blending method [54].

We make sure that the phase-field profile is sufficiently resolved by the grid, by requiring that the regularization length $\kappa$ is large enough compared with the grid spacing $h$, that is, $\kappa>2 h$. Because the crack path is not known a priori, we consider uniform grids, except in the regions where cracks cannot propagate; see Examples 2 and 3 in the following text. All the simulations consider an elastically isotropic material with Young's modulus $E=10^{9} \mathrm{~N} / \mathrm{m}^{2}$ and Poisson's ratio $v=0.3$.

We minimize the total energy in Equation (19) with respect to the displacement field and the phase-field following the alternate minimization algorithm described in [55]. At each load step, the energy is first minimized with respect to $\mathbf{u}$ holding $\phi$ fixed and then minimized with respect to $\phi$ holding $\mathbf{u}$ fixed. This procedure is iterated until convergence. This algorithm is particularly convenient in our examples. Because we only consider the ECH model with the tensor $\gamma_{i j k l}$, each one of the minimization steps involves a quadratic functional and hence the solution of a linear system. The minimization of the total energy can be performed with a variety of methods, including a monolithic Newton-type method. It is important to bear in mind that the staggered minimization process, as most optimization algorithms, leads in general to local minimizers and could even lead to saddle points of the total energy.

\section{RESULTS}

We now demonstrate through representative numerical simulations the ability of the proposed model to capture nontrivial crack patterns in brittle materials with strongly anisotropic surface energy.

\subsection{Example 1: Crack propagation direction as a function of material orientation}

We consider first a square domain with boundary conditions that promote the nucleation of a crack at the center of the left side of the domain, as depicted in Figure 3(a). The material used for the 


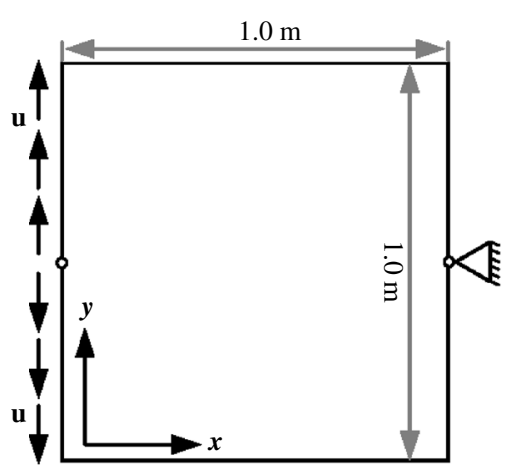

(a)

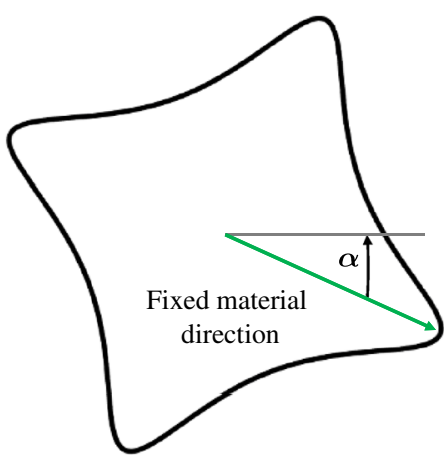

(b)

Figure 3. Example 1: geometry and boundary condition (a) and polar plot of the reciprocal surface energy (b), exhibiting strong anisotropy. The green arrow indicates a fixed material direction (one of the weakest directions), and $\alpha$ denotes the angle between the fixed material direction and the $x$-axis.

simulations has cubic symmetry and strongly anisotropic fracture surface energy. The surface energy parameters $\left(\bar{G}_{c}=500.0 \mathrm{~N} / \mathrm{m}, \gamma_{11}=19.0, \gamma_{12}=-18.0, \gamma_{44}=0.5\right)$ are chosen so that the maximum and minimum of $G_{c}(\theta)$ are 1157.5 and $707.1 \mathrm{~N} / \mathrm{m}$, respectively. The model is discretized with $200 \times 200$ uniformly distributed control points and the regularized length scale parameter $\kappa$ is set to $0.01 \mathrm{~m}$. Fixing $\kappa=0.01 \mathrm{~m}$, we observe no dependence of the results when the mesh is refined. The polar plot of the reciprocal surface energy is shown in Figure 3(b), where $\alpha$ denotes the angle between a fixed material direction and the $x$-axis. For materials with isotropic surface energy, the symmetry of the surface energy and of the boundary conditions imposes a crack propagation along the $x$-axis. In contrast, for a material with anisotropic surface energy, the crack path will emerge from a competition between elastic energy release rate maximization and surface energy minimization and therefore will in general deviate from the $x$ direction.

We analyze next how the crack direction changes as we change the material orientation. When the fixed material direction represented by the green arrow, a weak direction, coincides with the $x$-axis, the crack will propagate along this axis (Figure 4(a) and (b)). This situation is schematically shown in Figure 4(c), where the black line, representing material orientation, and red line, representing the crack propagation direction, are collinear. When the fixed material direction is rotated clockwise by an angle $\alpha\left(\alpha \leqslant 45^{\circ}\right)$ with respect to the $x$-axis, the crack orientation also rotates clockwise by an angle $\theta$ relative to the $x$-axis (Figure 4(d) and (e)). The schematic representation of this situation is shown in Figure 4(f). The sum of these signed angles $\alpha+\theta$, that is, the deviation of the red segment from the horizontal, quantifies the crack deviation from the weakest material direction. When the fixed material direction is further rotated clockwise $\left(\alpha \geqslant 45^{\circ}\right)$, the crack no longer follows the clockwise rotation of the material but rather finds an energetically favorable configuration by rotating counter-clockwise by a smaller amount (Figure 4(g-i)). Following the analogy of Wulff's construction [7], this behavior can be understood by noting that the preferred crack direction is given by the first tangency point of the polar plots in Figure 4(b, e, and h) with a vertical line moving leftwards toward the polar plot. Because this analogy is only an approximation in the present setting, the red points denoting the actual crack orientations slightly deviate from the tangency points just described.

We analyze next the systematic dependence of the crack propagation direction as a function of material orientation and visualize it with the previously introduced schematic representation as a rosace plot in Figure 5(a). This figure clearly highlights the four sectors of forbidden crack directions, as in the experiments in [7]. The polar plot of the reciprocal surface energy is shown in Figure 5(b), where the black line indicates the directions of observed cracks and the red line indicates the crack directions never observed as we continuously rotate the material orientation. The gray line is the double-tangent construction forming the convex-hull of the plot, as in the 


\section{Phase field}

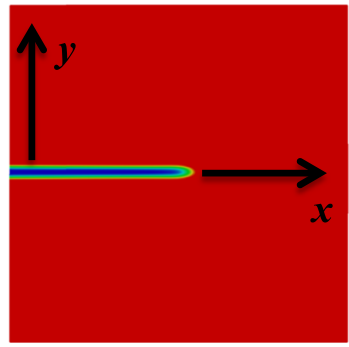

(a)

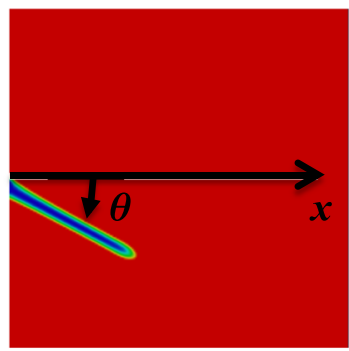

(d)

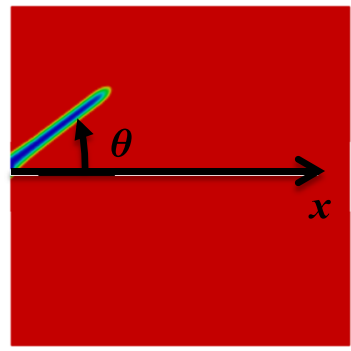

(g)
Material orientation polar plot of $1 / \mathrm{G}_{\mathrm{c}}$

(b)
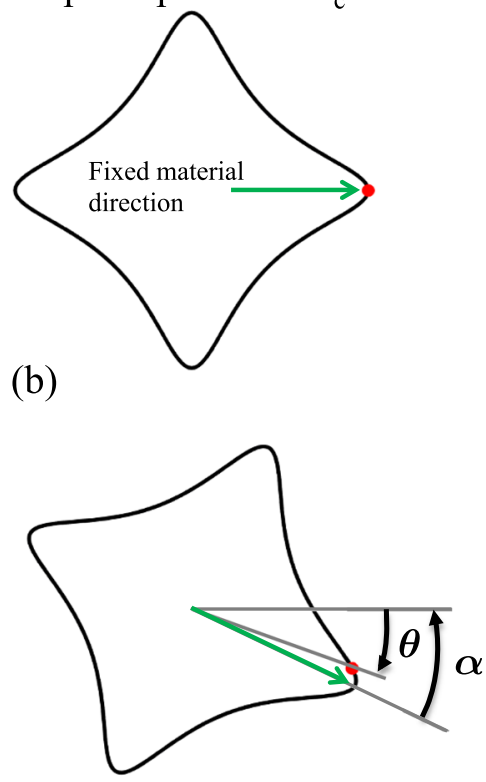

(e)

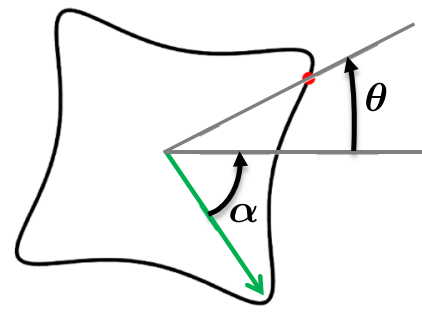

(h)
Schematic representation of material and crack orientation

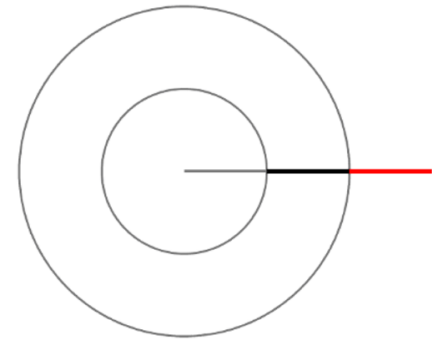

(c)

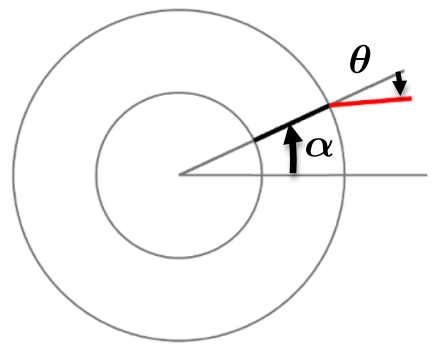

(f)

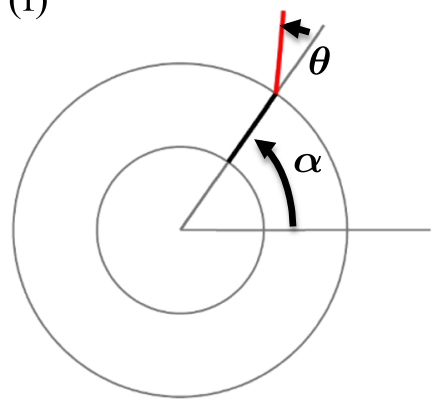

(i)

Figure 4. Example 1, as the material orientation is rotated relative to the sample geometry (top to bottom), $\alpha$ denotes the material orientation and $\theta$ the crack orientation, both relative to the $x$-axis aligned with the specimen. The first column shows the phase-field for a material oriented as shown in the second column, representing the polar plot of the reciprocal surface energy. The right column shows a schematic polar representation of the crack orientation (red segments, weak directions are vertical and horizontal) and the material orientation (black segment).

classical Maxwell construction [56]. The inset shows that there are observed crack directions inside of the convex-hull of the polar plot, suggesting that a mere convexification of the energy may yield a poor effective model. The regions where cracks are observed seem to agree well with the local stability condition given by the stiffness in Equation (25) and graphically determined by the regions of the polar plot with positive curvature. The experiments in [7] are consistent with this behavior suggesting a local MERR principle as discussed in Section 1, which in the simulations may be related to the trapping of the algorithm at local minima. However, we find that the point separating observed and forbidden directions, that is, separating the black from the red portions of the curve, does not coincide with the point where the stiffness of the surface energy (Equation (25)) changes sign. The point where $S(\theta)=0$ is marked with a cross in the inset of Figure 5(b). This difference may be due to inaccuracies in the numerical estimation of the crack direction or to the effect of boundary conditions. In any case, it deserves further scrutiny. 


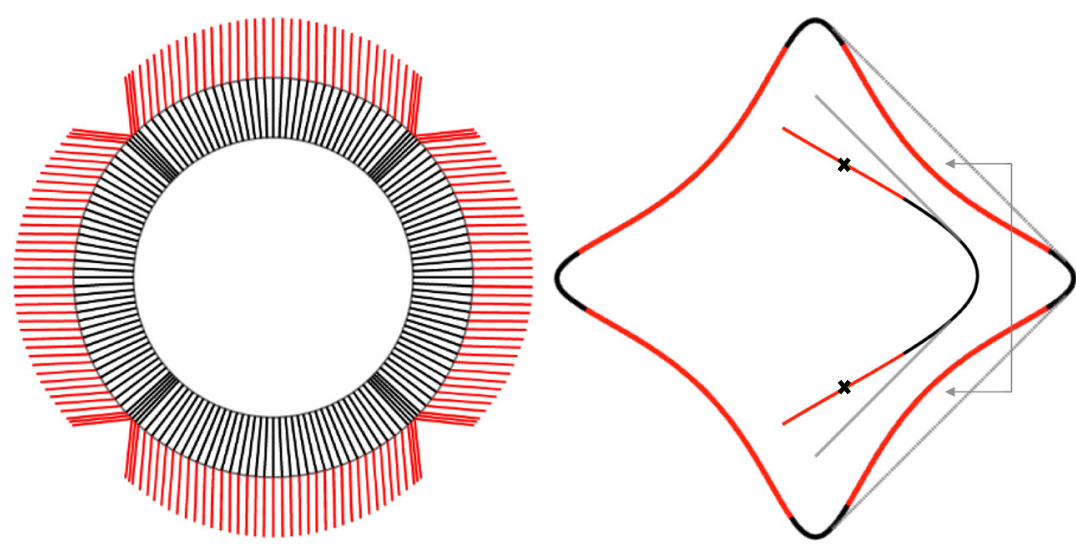

(a)

(b)

Figure 5. Systematic dependence of crack propagation (red segments) with material orientation (black segments), represented as a rosace with the convention of the previous figure (a). The polar plot of the reciprocal surface energy is represented in (b) and color coded depending on whether a given orientation is ever observed (black) or not (red) as we continuously rotate the material orientation. The inset shows that there are observed crack directions within the convex-hull of the polar plot. The region of observed cracks appears to correspond with the region of positive surface energy stiffness $S(\theta)$ (positive curvature of the polar plot)

(Equation (25)).

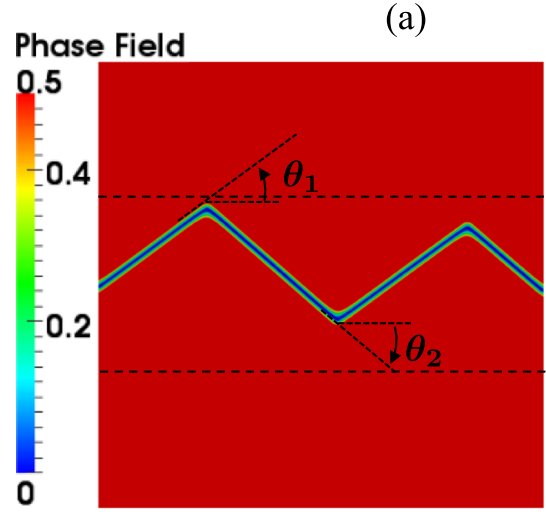

(b)
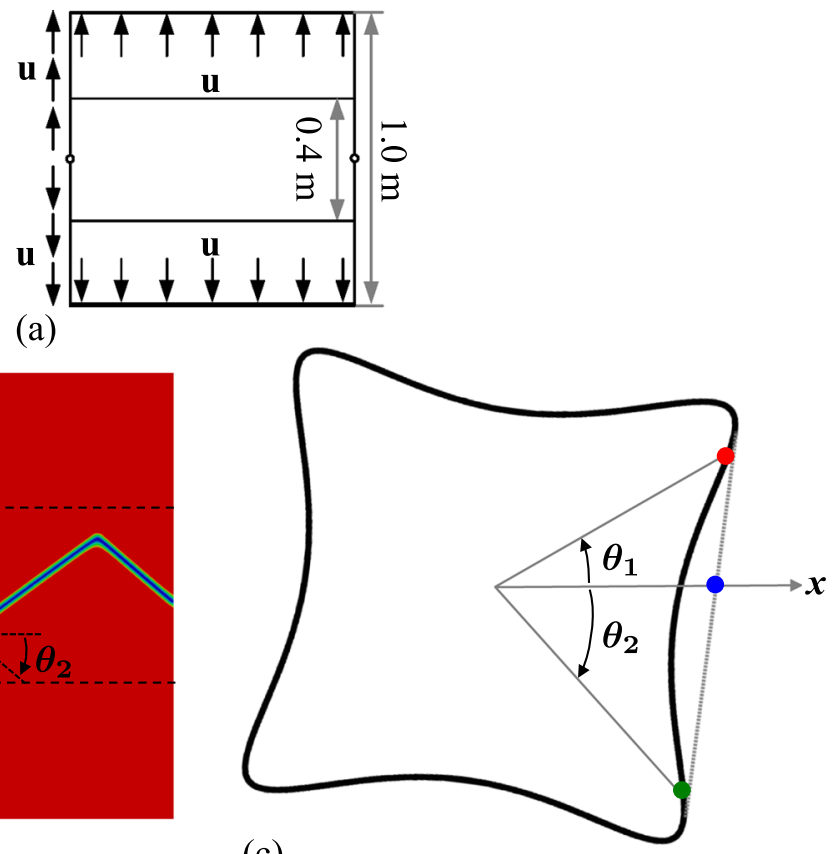

(c)

Figure 6. Example 2: crack propagation guided along a forbidden direction. (a) Computational model with boundary conditions. The displacement field at top and bottom bands is fully constrained. (b) Zigzag crack path obtained when the crack is guided along a forbidden direction. (c) The polar plot of the reciprocal surface energy and the double-tangent construction.

\subsection{Example 2: Zigzag crack paths}

In the presence of strong surface energy anisotropy, we analyze now how a crack propagates when the boundary conditions constrain the crack propagation along a forbidden direction. In the present example, we fully constrain the displacement field in the upper and lower bands of the domain 
as shown in Figure 6(a) to guide the crack as in the groove of a double cantilever beam crack propagation experiment [57]. A similar crack-guiding device has been implemented in [7] with tougher adhesive tapes. The surface energy parameters are the same as in Example 1, and $\kappa$ is set to $0.005 \mathrm{~m}$. The domain is discretized with a spacing of $1 / 400 \mathrm{~m}$ in a central band of $0.5 \mathrm{~m}$ height, while the grid is coarser in the upper and lower parts of the domain.

Figure 6(b) shows that, as in Example 1, the system initially chooses crack direction close to a weak direction. However, as the crack feels the presence of the constrained region but before touching it, it sharply turns to adopt a distant weak orientation that drives it apart from the obstacle. Interestingly, this new crack segment with angle $\theta_{2}$ turns upwards further apart from the constrained band in the lower part of the domain. Analogously, the third and last kinking event occurs before the crack reaches the upper constrained band. The kinking events are presumably the result of a more favorable elastic energy release rate as the crack deviates from the straight horizontal trajectory and approaches a mechanically constrained region. There is probably an energy cost associated to crack bending, here sharp kinking, implicit in the model and due to the second derivatives in the phasefield in the surface energy. Therefore, the elastic energy release rate incentive to kinking presumably needs to overcome this energetic penalty to sharply turning crack direction. Figure 6(c) shows the representation of the two crack orientations in the polar plot. In an effective model with a convexified surface energy, there are no forbidden directions and, if guided, the crack will propagate along the $x$-axis (blue point) with an energy cost given by the double-tangent construction, here $972.5 \mathrm{~N} / \mathrm{m}$. We compare this energy with the average surface energy per projected length along $x, 1008.5 \mathrm{~N} / \mathrm{m}$ to find a reasonable agreement. One of the factors that may explain the difference is, again, the energetic cost of kinks implicit in the model, which we have not explored so far.

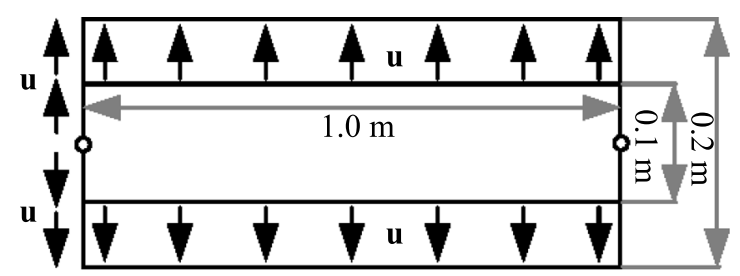

(a)

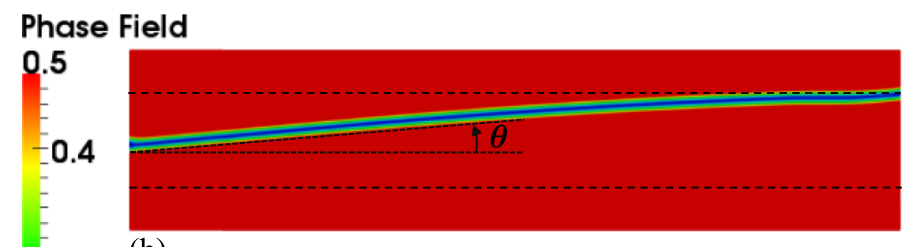

(b)

\section{2}

(c)
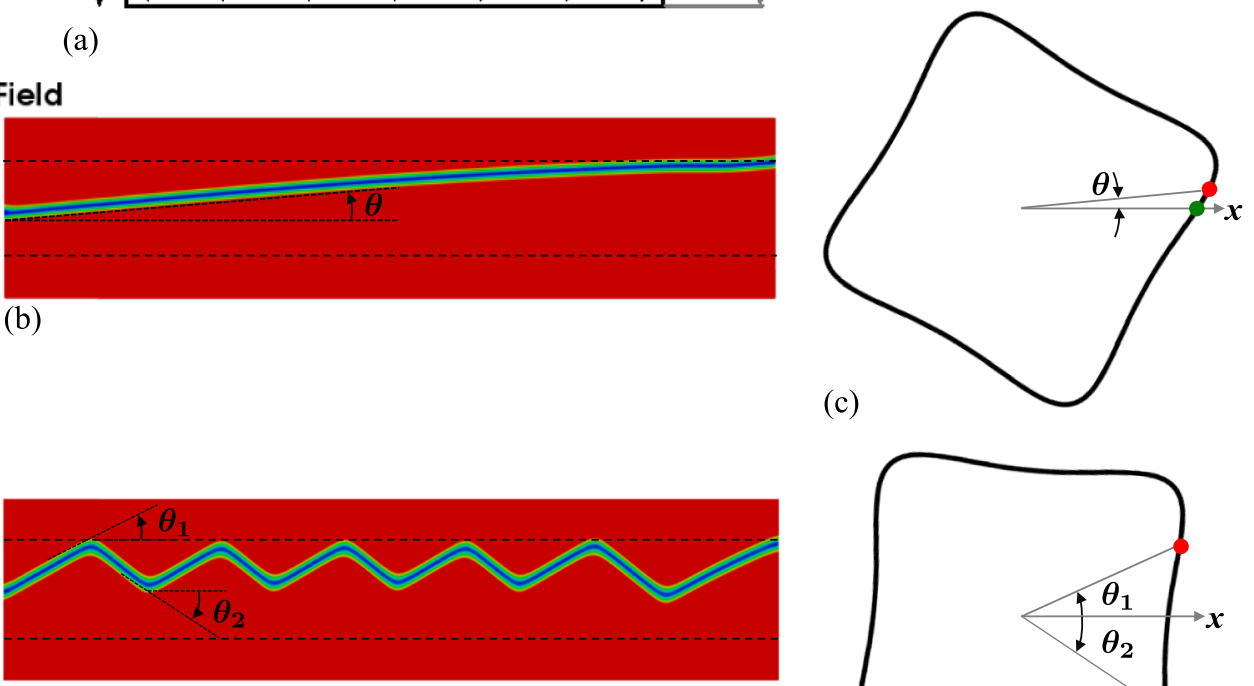

(d)

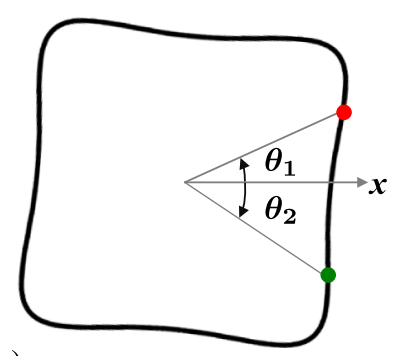

(e)

Figure 7. Example 3: crack propagation guided along an allowed but high-energy direction (b, c) or along a forbidden direction $(\mathrm{d}, \mathrm{e})$. The red and green dots in (c) represent the initial and final crack orientation, while in (e) represent the two orientations of the sawtooth pattern. 


\subsection{Example 3}

Inspired by the experiments in [7], we consider now a longer domain with similar boundary conditions to Example 2, as shown in Figure 7(a). We consider here a slightly different surface energy $\left(\bar{G}_{c}=500.0 \mathrm{~N} / \mathrm{m}, \gamma_{11}=17.0, \gamma_{12}=-15.0, \gamma_{44}=2.0\right)$ so that the maximum and minimum of $G_{c}(\theta)$ are 1131.7 and $826.4 \mathrm{~N} / \mathrm{m}$. As before, a central strip $0.15 \mathrm{~m}$ high has uniformly distributed points with a small node spacing of $1 / 400 \mathrm{~m}$, while the remainder of the domain has a coarser grid. The regularized length scale parameter $\kappa$ is set to $0.005 \mathrm{~m}$. As before, we expect that the crack will initially deflect away from the $x$-axis toward a weak direction. However, as shown in Figure 7(b), if the boundary of the guide (here parallel to $x$ ) is not a forbidden direction (Figure 7(c)) rather than kinking to adopt a weak direction, the crack runs along a direction of relatively large surface energy. In contrast, if the direction of the guide is a forbidden direction, as in Figure 7( $\mathrm{d}$ and e), the crack adopts a sawtooth configuration as in Example 2. Now, the upper kinking points are precisely at the upper boundary of the crack guide, while the lower kinks are very far apart from the lower boundary of the guide. As before, the location of these kinks emerges from the competition of elastic energy release rate, surface energy, and possibly kinking energy. The essential phenomenology of these two calculations has been reported experimentally in [7].

\section{CONCLUSIONS}

The fracture behavior of materials with strongly anisotropic surface energy is very important in many applications and leads to very interesting physics, including forbidden crack propagation directions, the possibility of guiding cracks along relatively high-energy directions, and sawtooth crack patterns. However, strongly anisotropic fracture had not been simulated computationally before to the best of our knowledge. This problem of fracture mechanics forces us to deeply interrogate fundamental questions such as the criteria to select the crack path. Recent theoretical [15] and experimental [7] studies favor a natural generalization of the MERR criterion. Previous phase-field models for weak anisotropy of the surface energy are consistent with this view and support a configurational torque balance [17] equivalent to MERR under certain conditions. The current work, presenting a variational phase-field model for materials with strongly anisotropic surface energy, provides a new tool to analyze this problem from the computational side and may be a starting point for the mathematical analysis of this problem. From a computational viewpoint, phase-field models appear as the best approach to investigate this complex problem where the crack orientation selection is so crucial. The variational nature of the model suggests that it obeys a MERR principle to select crack paths, and because we obtain crack propagation directions within the convex-hull of the polar plot of the reciprocal surface energy, the MERR principle appears to rely on local maximization, as also suggested in [7]. We are planning further studies to closely examine these issues.

To formulate the phase-field model for strongly anisotropic fracture, we have combined the classical variational phase-field model of brittle fracture [27] with the ECH framework [40, 41], proposed in the context of phase-field models of crystal growth. The result is a fourth-order model, because the energy functional involves the Hessian of the phase-field. Consequently, its numerical implementation by direct Galerkin methods requires smooth basis functions. Here, we resort to LME approximants, a family of smooth meshfree basis functions. We present a selected set of numerical examples that illustrate the main features of strongly anisotropic crack propagations. Our results reproduce many of the experimental observations in [7].

Our work is only a first step in the modeling and simulation of strongly anisotropic fracture using phase-field models, and many questions arise. From a theoretical viewpoint, we would like to understand the relation between our proposed model and a sharp-interphase crack propagation direction. We also plan to investigate the energetic penalty for crack bending implicit in the phase-field model, which presumably imposes an energy cost to crack kinking. It is not clear at this point how this contribution depends on the tensors $\alpha_{i j k l}, \beta_{i j k l}$, and $\gamma_{i j k l}$ of the ECH framework, and more importantly, it is not clear either if such a penalty has a physical meaning. Another set of interesting questions revolves around the modeling capabilities of the ECH framework, including the symmetries that can be described with such models, or the kinds of angle dependence of $G_{c}(\theta)$ that can 
be achieved, also including the tensors $\alpha_{i j k l}$ and $\beta_{i j k l}$ neglected here. A very simple motivation for this are geological materials, which typically exhibit twofold strong anisotropy. These tensors in the $\mathrm{ECH}$ model could be made dependent on the phase-field, and the surface energy may depend on the form of the dissipation function $f_{0}(\phi)$. All these extra features in the model introduce further nonlinearity in the equations and may require different numerical solution methods. It is also interesting to examine if one can model surface energies such that the polar plot of $1 / G_{c}(\theta)$ exhibits cusps, as one expects in brittle crystals with cleavage planes. Finally, we plan to extend this work to $3 \mathrm{D}$.

\section{APPENDIX A: ROTATION OF MATERIAL ORIENTATION RELATIVE TO THE SAMPLE}

For cubic symmetry and centrosymmetric materials, the fourth-rank tensors $\alpha_{i j k l}, \beta_{i j k l}$, and $\gamma_{i j k l}$ have the same major and minor symmetries as the anisotropic elastic stiffness tensor. We summarize next how to transform these tensors to a coordinate system that does not coincide with the principal material axes. The basis change formulae for fourth-rank tensors in Voigt notation are not straightforward. Suppose that the components of the fourth-rank tensor are given in basis $E$ and we want to determine its components in a second basis $\tilde{E}$. The change of basis formula for the tensor expressed as a matrix in Voigt notation $\mathbf{C}$ can be expressed in matrix form as

$$
\mathbf{C}^{\tilde{E}}=\mathbf{K} \mathbf{C}^{E} \mathbf{K}^{T}
$$

where $\mathbf{K}$ is a transformation matrix [58]. In 3D, when we rotate the material about the third coordinate vector by an angle $\Theta$, the transformation matrix reduces to

$$
\mathbf{K}=\left[\begin{array}{cccccc}
c^{2} & s^{2} & 0 & 0 & 0 & 2 c s \\
s^{2} & c^{2} & 0 & 0 & 0 & -2 c s \\
0 & 0 & 1 & 0 & 0 & 0 \\
0 & 0 & 0 & c & -s & 0 \\
0 & 0 & 0 & s & c & 0 \\
-c s & c s & 0 & 0 & 0 & c^{2}-s^{2}
\end{array}\right]
$$

where $c=\cos \Theta, s=\sin \Theta$. Consequently, in $2 \mathrm{D}$, the transformation matrix for the $3 \times 3$ Voigt representation of the fourth-order tensor (Equation. (17)) is just

$$
\mathbf{K}=\left[\begin{array}{ccc}
c^{2} & s^{2} & 2 c s \\
s^{2} & c^{2} & -2 c s \\
-c s & c s & c^{2}-s^{2}
\end{array}\right]
$$

\section{ACKNOWLEDGEMENTS}

We acknowledge the support of the Spanish Ministry of Science and Competitiveness through grant number DPI2011-26589. Bin Li gratefully acknowledges the financial supports from the China Scholarship Council. $\mathrm{CP}$ acknowledges FPU grant from the Spanish Ministry of Science and Competitiveness.

\section{REFERENCES}

1. Stroh A. Dislocations and cracks in anisotropic elasticity. Philosophical Magazine 1958; 3(30):625-646.

2. Barnett D, Asaro R. The fracture mechanics of slit-like cracks in anisotropic elastic media. Journal of the Mechanics and Physics of Solids 1972; 20(6):353-366.

3. Gao HJ, Chiu CH. Slightly curved or kinked cracks in anisotropic elastic solids. International Journal of Solids and Structures 1992; 29(8):947-972.

4. Argon AS, Qiao Y. Cleavage cracking resistance of large-angle grain boundaries in Fe-3 wt\% Si alloy. Philosophical Magazine A 2002; 82(17-18):3333-3347. 
5. Pérez R, Gumbsch P. Directional anisotropy in the cleavage fracture of silicon. Physical Review Letters 2000; 84(23):5347.

6. Riedle J, Gumbsch P, Fischmeister HF. Cleavage anisotropy in tungsten single crystals. Physical Review Letters 1996; 76(19):3594.

7. Takei A, Roman B, Bico J, Hamm E, Melo F. Forbidden directions for the fracture of thin anisotropic sheets: an analogy with the Wulff plot. Physical Review Letters 2013; 110:144 301.

8. Donath FA. Experimental study of shear failure in anisotropic rocks. Geological Society of America Bulletin 1961; 72(6):985-989.

9. Duveau G, Shao JF, Henry JP. Assessment of some failure criteria for strongly anisotropic geomaterials. Mechanics of Cohesive-Frictional Materials 1998; 3(1):1-26.

10. Niandou H, Shao JF, Henry JP, Fourmaintraux D. Laboratory investigation of the mechanical behaviour of tournemire shale. International Journal of Rock Mechanics and Mining Sciences 1997; 34(1):3-16.

11. Nasseri MHB, Mohanty B. Fracture toughness anisotropy in granitic rocks. International Journal of Rock Mechanics and Mining Sciences 2008; 45(2):167-193.

12. Khan AA, Vincent JFV. Anisotropy in the fracture properties of apple flesh as investigated by crack-opening tests. Journal of Materials Science 1993; 28(1):45-51.

13. Marder M. Cracks cleave crystals. EPL (Europhysics Letters) 2004; 66(3):364.

14. Gurtin ME, Podio-Guidugli P. Configurational forces and a constitutive theory for crack propagation that allows for kinking and curving. Journal of the Mechanics and Physics of Solids 1998; 46(8):1343-1378.

15. Chambolle A, Francfort GA, Marigo JJ. When and how do cracks propagate? Journal of the Mechanics and Physics of Solids 2009; 57(9):1614-1622.

16. Hakim V, Karma A. Crack path prediction in anisotropic brittle materials. Physical Review Letters 2005; 95(23):235 501.

17. Hakim V, Karma A. Laws of crack motion and phase-field models of fracture. Journal of the Mechanics and Physics of Solids 2009; 57(2):342-368.

18. Azhdari A, Nemat-Nasser S, Rome J. Experimental observations and computational modeling of fracturing in an anisotropic brittle crystal (sapphire). International Journal of Fracture 1998; 94(3):251-266.

19. Griffith AA. The phenomena of rupture and flow in solids. Philosophical Transactions of the Royal Society of London. Series A, Containing Papers of a Mathematical or Physical Character 1921; 221(582-593):163-198.

20. Freund LB. Dynamic Fracture Mechanics. Cambridge University Press: Cambridge, 1998.

21. Cotterell B. On brittle fracture paths. International Journal of Fracture Mechanics 1965; 1(2):96-103.

22. Goldstein RV, Salganik RL. Brittle fracture of solids with arbitrary cracks. International Journal of Fracture 1974; 10(4):507-523.

23. Wu CW. Maximum-energy-release-rate criterion applied to a tension-compression specimen with crack. Journal of Elasticity 1978; 8(2):235-257.

24. Sih GC. Strain-energy-density factor applied to mixed mode crack problems. International Journal of Fracture 1974; 10(3):305-321.

25. Erdogan F, Sih GC. On the crack extension in plates under plane loading and transverse shear. Journal of Basic Engineering 1963; 85:519.

26. Amestoy M, Leblond JB. Crack paths in plane situations-II. Detailed form of the expansion of the stress intensity factors. International Journal of Solids and Structures 1992; 29(4):465-501.

27. Bourdin B, Francfort GA, Marigo JJ. Numerical experiments in revisited brittle fracture. Journal of the Mechanics and Physics of Solids 2000; 48(4):797-826.

28. Arroyo M, Ortiz M. Local maximum-entropy approximation schemes: a seamless bridge between finite elements and meshfree methods. International Journal for Numerical Methods in Engineering 2006; 65(13):2167-2202.

29. Rosolen A, Millán D, Arroyo M. Second order convex maximum entropy approximants with applications to high order PDE. International Journal for Numerical Methods in Engineering 2013; 94(2):150-182.

30. Rosolen A, Peco C, Arroyo M. An adaptive meshfree method for phase-field models of biomembranes. Part I: approximation with maximum-entropy approximants. Journal of Computational Physics 2013; 249:303-319.

31. Francfort GA, Marigo JJ. Revisiting brittle fracture as an energy minimization problem. Journal of the Mechanics and Physics of Solids 1998; 46(8):1319-1342.

32. Bourdin B, Francfort G, Marigo JJ. The variational approach to fracture. Journal of Elasticity 2008; 91(1-3):5-148.

33. Bourdin B, Larsen C, Richardson C. A time-discrete model for dynamic fracture based on crack regularization. International Journal of Fracture 2011; 168(2):133-143.

34. Borden MJ, Hughes TJ, Landis CM, Verhoosel CV. A higher-order phase-field model for brittle fracture: formulation and analysis within the isogeometric analysis framework. Computer Methods in Applied Mechanics and Engineering 2014; 273:100-118.

35. Kobayashi R. Modeling and numerical simulations of dendritic crystal growth. Physica D: Nonlinear Phenomena 1993; 63(3):410-423.

36. Taylor J, Cahn J. Diffuse interfaces with sharp corners and facets: phase field models with strongly anisotropic surfaces. Physica D: Nonlinear Phenomena 1998; 112(3):381-411.

37. Eggleston JJ, McFadden GB, Voorhees PW. A phase-field model for highly anisotropic interfacial energy. Physica D: Nonlinear Phenomena 2001; 150(1):91-103.

38. Torabi S, Lowengrub J, Voigt A, Wise S. A new phase-field model for strongly anisotropic systems. Proceedings of the Royal Society A: Mathematical, Physical and Engineering Science 2009; 465(2105):1337-1359. 
39. Cahn JW, Hilliard JE. Free energy of a nonuniform system. I: interfacial free energy. The Journal of Chemical Physics 1958; 28(2):258-267.

40. Abinandanan TA, Haider F. An extended Cahn-Hilliard model for interfaces with cubic anisotropy. Philosophical Magazine A 2001; 81(10):2457-2479.

41. Torabi S, Lowengrub J. Simulating interfacial anisotropy in thin-film growth using an extended Cahn-Hilliard model. Physical Review E 2012; 85(4):041 603.

42. McKenna IM, Gururajan MP, Voorhees PW. Phase field modeling of grain growth: effect of boundary thickness, triple junctions, misorientation, and anisotropy. Journal of Materials Science 2009; 44(9):2206-2217.

43. Pham K, Amor H, Marigo JJ, Maurini C. Gradient damage models and their use to approximate brittle fracture. International Journal of Damage Mechanics 2011; 20(4):618-652.

44. Pham K, Marigo JJ. From the onset of damage to rupture: construction of responses with damage localization for a general class of gradient damage models. Continuum Mechanics and Thermodynamics 2013; 25(2-4):147-171.

45. Borden MJ. Isogeometric analysis of phase-field models for dynamic brittle and ductile fracture. Ph.D. Thesis, Computational Science, Engineering, and Mathematics, University of Texas at Austin, Austin, USA, August 2012.

46. Herring C. Some theorems on the free energies of crystal surfaces. Physical Review 1951; 82(1):87.

47. Müller P, Métois JJ. Anisotropy of the surface thermodynamic properties of silicon. Thin Solid Films 2008; 517(1):65-68.

48. Sekerka RF. Analytical criteria for missing orientations on three-dimensional equilibrium shapes. Journal of Crystal Growth 2005; 275(1):77-82.

49. Rosolen A, Millán D, Arroyo M. On the optimum support size in meshfree methods: a variational adaptivity approach with maximum entropy approximants. International Journal for Numerical Methods in Engineering 2010; 82(7):868-895.

50. Peco C, Rosolen A, Arroyo M. An adaptive meshfree method for phase-field models of biomembranes. Part II: a Lagrangian approach for membranes in viscous fluids. Journal of Computational Physics 2013; 249:320-336.

51. Scott M, Li X, Sederberg T, Hughes T. Local refinement of analysis-suitable T-splines. Computer Methods in Applied Mechanics and Engineering 2012; 213-216:206-222.

52. Vuong AV, Giannelli C, Jüttler B, Simeon B. A hierarchical approach to adaptive local refinement in isogeometric analysis. Computer Methods in Applied Mechanics and Engineering 2011; 200(49-52):3554-3567.

53. Wang W, Zhang Y, Liu L, Hughes TJR. Trivariate solid T-spline construction from boundary triangulations with arbitrary genus topology. Computer-Aided Design 2013; 45:351-360.

54. Rosolen A, Arroyo M. Blending isogeometric analysis and local maximum entropy meshfree approximants. Computer Methods in Applied Mechanics and Engineering 2013; 264:95-107.

55. Bourdin B. Numerical implementation of the variational formulation for quasi-static brittle fracture. Interfaces and Free Boundaries 2007; 9(3):411-430.

56. Huang K. Lectures on Statistical Physics and Protein Folding. World Scientific: Singapore, 2005.

57. Wu CCM, McKinney KR, Rice RW. Zig-zag crack propagation in $\mathrm{MgAl}_{2} \mathrm{O}_{4}$ crystals. Journal of Materials Science Letters $1995 ;$ 14(7):474-477.

58. Ting TCT. Anisotropic Elasticity: Theory and Applications. Oxford University Press: New York, 1996. 